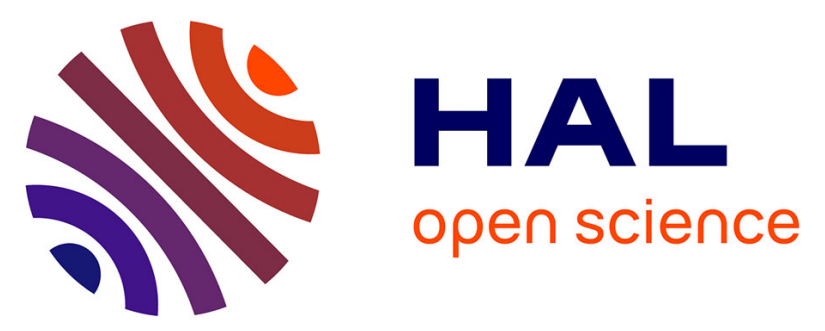

\title{
3-oxo-C12:2-HSL, Quorum Sensing molecule from human intestinal microbiota, inhibits proinflammatory pathways in immune cells via bitter taste receptors
}

Garance Coquant, Doriane Aguanno, Loic Brot, Christine Belloir, Julie Delugeard, Nathalie Roger, Hang-Phuong Pham, Loïc Briand, Marielle Moreau, Luisa de Sordi, et al.

\section{To cite this version:}

Garance Coquant, Doriane Aguanno, Loic Brot, Christine Belloir, Julie Delugeard, et al.. 3-oxoC12:2-HSL, Quorum Sensing molecule from human intestinal microbiota, inhibits proinflammatory pathways in immune cells via bitter taste receptors. 2022. hal-03546045

\author{
HAL Id: hal-03546045 \\ https://hal.science/hal-03546045
}

Preprint submitted on 27 Jan 2022

HAL is a multi-disciplinary open access archive for the deposit and dissemination of scientific research documents, whether they are published or not. The documents may come from teaching and research institutions in France or abroad, or from public or private research centers.
L'archive ouverte pluridisciplinaire HAL, est destinée au dépôt et à la diffusion de documents scientifiques de niveau recherche, publiés ou non, émanant des établissements d'enseignement et de recherche français ou étrangers, des laboratoires publics ou privés. 


\section{Research Square

\section{3-oxo-C12:2-HSL, Quorum Sensing Molecule from Human Intestinal Microbiota, Inhibits Pro- inflammatory Pathways in Immune Cells via Bitter Taste Receptors}

\section{Garance Coquant}

Sorbonne University

Doriane Aguanno

École Pratique des Hautes Études

Loic Brot

Sorbonne University

Christine Belloir

Centre des Sciences du Goût et de l'Alimentation

Julie Delugeard

Sorbonne University

Nathalie Roger

Sorbonne University

Hang-Phuong Pham

Parean Biotechnology

Loic Briand

Centre des Sciences du Goût et de l'Alimentation

Marielle Moreau

LVMH (France)

Luisa De Sordi

Sorbonne University

Véronique Carrière

Sorbonne University

Jean-Pierre Grill

Sorbonne University

Sophie Thenet

École Pratique des Hautes Études

Philippe Seksik ( $\square$ philippe.seksik@aphp.fr)

Sorbonne University 


\section{Research Article}

Keywords: 3-oxo-C12:2-HSL, Quorum Sensing molecule, human intestinal microbiota, pro-inflammatory pathways, immune cells

Posted Date: January 5th, 2022

DOI: https://doi.org/10.21203/rs.3.rs-1191781/v1

License: (c) (i) This work is licensed under a Creative Commons Attribution 4.0 International License.

Read Full License 


\section{Abstract}

In the gut ecosystem, microorganisms regulate group behaviour and interplay with the host via a molecular system called quorum sensing (QS). The QS molecule 3-oxo-C12:2-HSL, first identified in human gut microbiota, exerts anti-inflammatory effects and could play a role in inflammatory bowel diseases where dysbiosis has been described. Our aim was to identify which signalling pathways are involved in this effect. We observed that 3-oxo-C12:2-HSL decreases expression of pro-inflammatory cytokines such as, Interleukine-1 $\beta$ (-3 \%) and Tumor Necrosis Factor-a (TNFa) (40 \%) by stimulated immune RAW264.7 cells and decreased TNF secretion by stimulated PBMC in a dose-dependent manner, between $25 \mu \mathrm{M}$ to $100 \mu \mathrm{M}$. Transcriptomic analysis of RAW264.7 cells exposed to 3-oxo-C12:2-HSL, in a pro-inflammatory context, highlighted JAK-STAT, NF-KB and TFN signalling pathways and we confirmed that 3-oxo-C12:2-HSL inhibited JAK1 and STAT1 phosphorylation. We also showed through a screening assay that 3-oxo-C12:2-HSL interacted with several human bitter taste receptors. Its anti-inflammatory effect involved TAS2R38 as shown by pharmacologic inhibition and led to an increase in intracellular calcium levels. We thus unravelled the involvement of several cellular pathways in the anti-inflammatory effects exerted by the QS molecule 3-oxo-C12:2-HSL.

\section{Introduction}

Inflammatory bowel disease (IBD), including Crohn's disease (CD) and ulcerative colitis (UC), are chronic relapsing inflammatory conditions of the gastrointestinal tract leading to bowel damages and increased risk $^{1}$ of intestinal cancer. The incidence and prevalence of $C D$ and $U C$ is increasing and an estimated $0.3 \%$ of the European population suffers from IBD, representing 2.5 to 3 million people. IBD, which affects young adults and is long-lasting, accounts for substantial costs to the health care system and society. Disease management has changed significantly over the last decade with the use of parenterally administrated biologic agents and new small oral molecules such as pan-Janus kinase (JAK) inhibitor ${ }^{3}$. Immunosuppressants are used more widely and earlier in the progression of the disease. However, this strategy is not always effective, is quite expensive and exposes patients to adverse effects, mainly serious infections and malignancies ${ }^{4}$. Thus, there is both a need and a place for more "gut restricted" and physiological therapeutic approaches mostly based on a better knowledge of the gut microbiota.

As a matter of fact, gut microbiota has been strongly implicated in the pathogenesis of IBD $^{5,6}$ where microbial unbalance (dysbiosis) has been described ${ }^{7}$. Thus microbiota-based treatments, such as faecal microbiota transplantation, are currently being studied ${ }^{8,9}$. In this setting, investigating gut microbiotaderived molecules remains an attractive strategy to control gut inflammation and prevent IBD flares. In fact, gut microbiota reciprocally interacts with co-evolved host epithelial and immune cells. This results in a beneficial mutualistic relationship in which host-microbiota interactions drive inflammatory responses as dysbiosis alters microbial metabolites production ${ }^{10-13}$. A largely overlooked metabolite component in the intestinal context is that of diffusible signal compounds represented by bacterial quorum sensing (QS) molecules, called autoinducers ${ }^{14}$. Acyl-Homoserine Lactones (AHL) are a class of autoinducers that 
can impact the human host, as part of the inter-kingdom signalling ${ }^{14}$. Our team identified for the first time several AHLs in the gut ecosystem, and among them, the most prominent was the 3-oxo-C12:2-HSL, a molecule that had never been described ${ }^{15}$. 3-oxo-C12:2-HSL was decreased in IBD patient compared to healthy subjects and its presence was correlated with normobiosis ${ }^{15}$. 3-oxo-C12:2-HSL is structurally close to 3-oxo-C12-HSL synthesized by Pseudomonas aeruginosa, a molecule whose effects on host cells are well reported in the literature. We previously described the anti-inflammatory and dose-dependent effects of 3-oxo-C12:2-HSL on intestinal epithelial cells Caco-2/TC7 stimulated by interleukin- $\beta^{15}$. In addition, we have demonstrated that 3-oxo-C12:2-HSL is protective of pro-inflammatory cytokine-induced tight junctions disruption and thus has a beneficial role on intestinal barrier function ${ }^{16}$. In the present study, we aim to characterise the effects of 3-oxo-C12:2-HSL on immune cells and to identify cellular targets of the autoinducer. Using the macrophage cell line RAW264.7 as well as the peripheral blood mononuclear cell (PBMC) stimulated by lipopolysaccharides (LPS) and interferon-y (IFNy), we assessed how 3-oxo-C12:2-HSL modulates host-inflammatory response and the mechanisms involved. Our results show that this AHL exerts anti-inflammatory effects by down-regulating pro-inflammatory cytokines secretion. Using transcriptomic analysis, we have identified several pathways involved, one of them being the JAK-STAT pathway. 3-oxo-C12:2-HSL is able to prevent RAW264.7 macrophages from JAK-STAT signalling activation induced by LPS and IFNy. We also identify bitter taste receptors as potential membrane receptors for this AHL.

\section{Results}

\section{3-oxo-C12:2-HSL reduces expression and secretion of pro- inflammatory cytokines by activated immune cells}

We first observed the effect of 3-oxo-C12:2-HSL on cytokine secretion by immune cells. We exposed RAW264.7 murine macrophages cells to lipopolysaccharides (LPS) and Interferon-y (IFNy) to trigger classical activation of M1 macrophages ${ }^{17}$. Cells were treated with or without $50 \mu \mathrm{M}$ of 3-0xo-C12:2-HSL followed by analysis of cytokines levels in the supernatant (Fig. 1a, b, c). Lower secretion of proinflammatory Tumor Necrosis Factor-a (TNFa) -40 \%, Fig. 1a) and Interleukine-1 $($ IL-1 $\beta$ (-35 \%, Fig. 1b) was observed in presence of 3-oxo-C12:2-HSL compared with the control. We then investigated the effects on inflammation at the transcript level. mRNA levels of the two cytokines mentioned above were reduced in activated macrophages exposed to 3-oxo-C12:2-HSL, to the same extent as the protein (Fig. 1c, d). Anti-inflammatory cytokine IL-10 expression was strongly increased when cells were exposed to the $\mathrm{AHL}$ (Fig. 1e).

To confirm these results in a more physiological model, we tested the impact of the AHL on Peripheral Blood Mononuclear Cells (PBMC) from healthy donors. Inflammation was triggered with LPS (10 ng/mL), and we exposed PBMC to an increasing dose of 3-oxo-C12:2-HSL. We showed that TNFa secretion was strongly decreased in a dose-dependent manner, between $25 \mu \mathrm{M}$ to $100 \mu \mathrm{M}$ (Fig. 1f). 
Of note, cytotoxicity was monitored through lactate dehydrogenase release for all experiments and there was no significant difference from the control for the doses of AHL used (Supplementary Fig. 1).

\section{3-oxo-C12:2-HSL modulates gene expression in RAW264.7 cells}

In an attempt to understand the mechanisms underpinning the immunomodulatory effects of 3-oxoC12:2-HSL on immune cells, we performed a transcriptomic analysis of the activated RAW264.7 cells exposed to 3-oxo-C12:2-HSL. We observed that 3-oxo-C12:2-HSL down-regulated 736 genes and upregulated 1140 genes compared with control cells treated with DMSO (Figure 2a).

Cells were stimulated with LPS and IFNy in absence (control) or in presence of $50 \mu \mathrm{M}$ 3-oxo-C12:2-HSL for 2 hours (a): Volcano plot of differentially expressed genes between the activated cells cultured in absence and in presence of 3-oxo-C12:2-HSL. Blue, grey and red dots represent down regulated, not significantly regulated and up regulated genes respectively. (b) Significant KEGG pathways involved in the inflammation process and differentially modulated by 3-oxo-C12:2-HSL were identified by EGSA method ( $p$ value $<0.01$ ). Red and green bars represent down-regulated and up-regulated pathways respectively. All significant modulated pathways are displayed in Supplementary Figure 2. (c) Significant Gene Ontology (GO) biology processes enriched by differentiated expressed genes modulated by 3-oxoC12:2-HSL. Terms were annotated using the Database for Annotation, Visualization and Integrated Discovery (DAVID). Red and green bars represent down-regulated and up-regulated pathways respectively. (GO: Gene Ontology, KEGG: Kyoto Encyclopedia of Genes and Genomes)

Differentially expressed genes were annotated by Gene Ontology (GO) analysis (Fig. 2c). GO analysis revealed that down-regulated genes were enriched in "immune system process", "innate immune response" and "inflammatory response" categories, in accordance with the anti-inflammatory effects of 3oxo-C12:2-HSL observed in Figure 1. In terms of molecular function, we identified that the downregulated genes were enriched in the NF-KB and Toll Like Receptor (TLR) and TNF signalling pathways. The latter correlated with the decrease of TNFa protein secretion observed in Figure 1a and $\mathrm{f}$.

A KEGG pathway analysis was carried out to classify and group differentially expressed genes in cellular processes (Fig. 2b). A large number of pathways were modulated by the AHL (Supplementary Fig. 2), among which, the most down-regulated pathways were "Cytokine-cytokine receptor interaction" and "TFN signalling pathway". A selection of pathways related to inflammation and IBD modulated by 3-oxo-C12:2HSL is displayed on Figure 2c. This includes crucial pathways directly involved in cell signalling during inflammation such as the "JAK-STAT signalling pathway", the "NF-KB signalling pathway" and the "TNF signalling pathway". This also includes pathways related to IBD such as "apoptosis" and "ABS transporters". Interestingly, 3-oxo-C12-HSL produced by P. aeruginosa was reported to up-regulate both apoptosis and expression of $A B C$ receptors ${ }^{18}$, suggesting that these two $A H L$ do not exert similar effects.

From both GO and KEGG pathway analyses, we were able to pinpoint specific cellular pathways involved in the signalling of 3-oxo-C12:2-HSL. We have chosen to study one lead specifically: the JAK-STAT 
signalling pathway, as it is a target of interest in new therapeutics developed against IBD ${ }^{19,20}$.

\section{3-oxo-C12:2 prevents the activation of the JAK-STAT pathway}

To explore the effects of 3-oxo-C12:2-HSL on the JAK-STAT pathway, we analysed the phosphorylation level of proteins involved in this pathway. As the latter is composed of a family of JAK and STAT proteins, we studied several of them in RAW264.7 macrophages activated by LPS and IFNY and exposed or not to 3-oxo-C12:2-HSL (Figure 3). When cells were treated with the pro-inflammatory cocktail, we observed an increase of the phosphorylation of JAK1, JAK2, STAT1, STAT3 proteins (Fig. 3a, b, c, d, e). When activated macrophages were exposed to 3-oxo-C12:2-HSL, a reduced phosphorylation level of JAK1 (-50\%) and STAT1 (-40\%) protein was observed, in comparison with activated cells without AHL treatment (Fig. 3a and b). However, JAK2 and STAT3 phosphorylation levels were not significantly altered upon exposure to 3-oxo-C12:2-HSL.

These results demonstrate that 3-oxo-C12:2-HSL prevents the activation of the JAK-STAT pathway, by specifically reducing the phosphorylation of JAK1 and STAT1 proteins.

Cells were stimulated with LPS and IFNy in absence (control) or in presence of $50 \mu \mathrm{M}$ 3-oxo-C12:2-HSL for 2 hours. The levels of phosphorylated proteins P-JAK1 (a), STAT1 (b), JAK2 (c), STAT3 (d) were normalized to their respective unphosphorylated forms. Results are expressed as mean \pm SEM from 3 independent experiments. One-Way ANOVA, Dunnett's post-test $* * P<0.01$, $* \star * P<0.001$, $* * \star * P<0.0001$ vs. control. (e) Reconstructed images from Simple Western analysis of protein levels and actin used as housekeeping protein are displayed. They are based on the area under the chemiluminescence signal curve obtained for one experiment, representative of 4 independent experiments performed in duplicates. Molecular markers are indicated on the left (KDa). 3oC12:2 stands for 3-oxo-C12:2-HSL.

\section{3-oxo-C12:2-HSL interacts with bitter taste receptors}

It was previously reported that the AHL 3-oxo-C12-HSL synthesized by $P$. aeruginosa might exert some of its effects through its interaction via the human bitter taste receptor TAS2R38 ${ }^{21,22}$. Bitter taste receptors are $\mathrm{G}$ protein-coupled receptor and their role beyond oral taste has been extensively studied throughout the last decade ${ }^{23}$. Taste receptors are all expressed along the digestive tract ${ }^{24-27}$, but also by immune cells $^{21,28,29}$. Based on the similarities in the chemical structure of 3-oxo-C12-HSL and 3-oxo-C12:2-HSL we adopted a targeted approach to identify potential eukaryotic receptors for intestinal 3-oxo-C12:2-HSL and hypothesized that 3-oxo-C12:2-HSL could also interact with the bitter taste receptor TAS2R38

We pre-exposed RAW264.7 cells to probenecid, a known allosteric inhibitor of TAS2R38 30,31 and its mouse orthologue TAS2R138 (Fig. 4a) then added LPS and IFNY and 3-oxo-C12:2-HSL. First, we noted that probenecid treatment had no effect on the TNFa secretion in activated cells cultured in absence of 3oxo-C12:2-HSL (control). When exposed to probenecid and 3-oxo-C12:2-HSL in a pro-inflammatory context, more TNFa was secreted compared to cells only exposed to 3-oxo-C12:2-HSL without inhibitor 
treatment. This result shows that when TAS2R138 is inhibited, the 3-oxo-C12:2-HSL anti-inflammatory effect is abolished.

To elucidate if 3-oxo-C12:2-HSL was able to activate human TAS2R38 and other bitter taste receptors, we screened 25 known human bitter taste receptors in HEK293T reporter-cells (Fig. 4b) ${ }^{32}$. Among these bitter taste receptors, the AHL was able to activate TAS2R1, TAS2R8, TAS2R10, TAS2R13, TAS2R14, TAS2R38. TAS2R8 $\left(E_{50}=333 \pm 27 \mu \mathrm{M}\right)$, TAS2R1 $\left(E_{50}=496 \pm 38 \mu \mathrm{M}\right)$ and TAS2R14 $\left(E_{50}=693 \pm 2 \mu \mathrm{M}\right)$ had the highest half maximal effective concentration $\left(\mathrm{EC}_{50}\right)$, translating the highest potency of 3-oxo-C12:2-HSL, while TAS2R10 $\left(E_{50}=438 \pm 120 \mu \mathrm{M}\right)$ and TAS2R38 $\left(E_{50}=476 \pm 125 \mu \mathrm{M}\right)$ exhibited a lower $E_{50}$ value. No response was detected at concentration below $250 \mu \mathrm{M}$.

Transduction of taste signalling results in the opening of IP3R-dependant calcium channels and the intracellular $\mathrm{Ca}^{2+}$ release ${ }^{23}$. Therefore, we investigated the effect of 3-oxo-C12:2-HSL with a Fluo-4 probe in the basal state, with the endoplasmic reticulum $\mathrm{Ca}^{2+}$ ATPase inhibitor thapsigargin as a positive control. We observed an increase in intracellular calcium level when RAW264.7 cells were exposed to 3oxo-C12:2-HSL compared with the control (Fig. $4 \mathrm{c}$ and d). The quantification of calcium response shows that in presence of 3-oxo-C12:2-HSL the slope of the curve was significantly higher than that observed in the control condition (Fig. 4d).

\section{Discussion}

As the human gut is one of the most densely populated microbial ecosystems, studying QS molecules, related to bacterial density, seems legitimate in this environment. AHLs are the most studied QS autoinducers and, using mass spectrometry, our team previously identified one that had never been documented before in the gut ecosystem, 3-oxo-C12:2-HSL ${ }^{15}$. Our initial work showed that 3-oxo-C12:2HSL correlated with normobiosis and was less prevalent in IBD associated dysbiosis. Our group observed that this molecule decreased IL-8 production in the intestinal epithelial cell line Caco-2/TC7 stimulated by $\mathrm{IL}-1 \beta^{15}$. We therefore sought to investigate how 3-oxo-C12:2-HSL can modulate inflammatory response of immune cells. Here, we demonstrate that 3-oxo-C12:2-HSL exerts anti-inflammatory effects on immune cells by preventing JAK-STAT pathway activation and by activating bitter taste signalling (Fig. 5).

In immune cells, 3-oxo-C12:2-HSL is able to activate bitter taste receptors, which are G-protein coupled receptors, triggering a signalling cascade resulting in the release of calcium from the endoplasmic reticulum. In addition, in murine activated macrophages the presence of 3-oxo-C12:2-HSL attenuates the activation of the JAK-STAT signalling pathways, by specifically preventing JAK1 and STAT1 phosphorylation. This leads to a decrease in pro-inflammatory cytokine secretions and an overall reduced inflammatory response. Part of the effects of 3-oxo-C12:2-HSL on inflammatory response is dependent on bitter taste receptors. Created with BioRender.com.

AHLs have been described to modulate host's immunity, in particular 3-oxo-C12-HSL from Pseudomonas aeruginosa, structurally close to 3-oxo-C12:2-HSL. A number of studies have reported that 3-oxo-C12-HSL 
downregulated cytokine secretion by macrophages ${ }^{33-35}$, dendritic cells ${ }^{36}$, T lymphocytes $^{33,37,38}$ as well as epithelial cells ${ }^{15,39}$. In the present study, the intestinal unsaturated 3-oxo-C12:2-HSL displays antiinflammatory effects by downregulating secretion of pro-inflammatory cytokine TNFa and IL-1 $\beta$, as well as their respective gene expression by RAW264.7 murine macrophages. Interestingly, this AHL was also able to increase anti-inflammatory cytokine IL-10 expression, suggesting a potent anti-inflammatory effect in immune cells. These findings were confirmed using a more physiological model, PBMC stimulated by LPS, in which TNFa was strongly decreased by 3-oxo-C12:2-HSL exposure, in a dosedependent manner. We activated RAW264.7 cells with LPS and IFNy, leading the macrophages to a M1 profile ${ }^{17}$, similar to what is observed during IBD physiopathology. Indeed, in the mucosa of patients, there is an influx of pro-inflammatory macrophages ${ }^{40}$. Under such conditions, activation of the JAK-STAT pathway is enhanced, with a particular involvement of the STAT1 protein ${ }^{41-43}$. One paper has reported that 3-oxo-C12-HSL is able to modulate the JAK-STAT pathway in breast carcinoma cells ${ }^{44}$, but our group is the first to report AHL modulation of this pathway in immune cells. The point of 3-oxo-C12:2-HSL was to prevent the phosphorylation of JAK1 and STAT1, which is particularly relevant in the field of IBD. Indeed, 3-oxo-C12:2-HSL, which is decreased in IBD patients ${ }^{15}$, appears as a microbial product able to modulate IBD pathways involved in chronic inflammation. Novel IBD treatments are targeting the JAK proteins ${ }^{20}$. Current IBD drugs target one or more cytokines (such as anti-TNF) ${ }^{20}$ while new oral small molecules act by inhibiting either pan-JAK or specific JAK pathways. However, clinical trials with JAK inhibitors revealed serious adverse events ${ }^{20}$. Alternative approaches using molecules targeting the gut are appealing, with drugs exerting a strong anti-inflammatory effect while being less prone to side effects. The use of $\mathrm{AHL}$ as modulators of gut inflammation is thus an interesting and promising path. Moreover, 3-oxo-C12:2-HSL was shown to stabilize tight junctions proteins involved in gut barrier ${ }^{16}$, thereby counterbalancing the disruption of the gut barrier in IBD ${ }^{45}$. Altogether, these results pave the way for future therapeutic development of this type of molecule. In this setting, it remains crucial to study the mechanisms of action by which a given LHA signals its effect on epithelial and immune cells. Our future studies will focus on the JAK-STAT pathway and explore how 3-oxo-C12:2-HSL is able to prevent phosphorylation of JAK1 and STAT1 proteins.

Interkingdom effects of $A H L$ raise the question of eukaryotic $A H L$ receptors. Some proteins were identified as target of 3-oxo-C12-HSL from P. aeruginosa: the scaffolding protein IQGAP1f, the aryl hydrocarbon receptor (AhR) ${ }^{46}, \mathrm{PPAR}^{47,48}$, the bitter taste receptor TAS2R3821,22,49,50. The latter has aroused our curiosity due to the growing interest in bitter taste receptors, particularly in their extra-oral expression ${ }^{23}$. In this study, we demonstrate that TAS2R38 signalling is needed for 3-oxo-C12:2-HSL to exert anti-inflammatory effects on immune cells. Taste receptor signalling, through the second messenger IP3, activates calcium release from the endoplasmic reticulum ${ }^{23}$. We demonstrated that 3-oxo-C12:2-HSL is able to induce calcium flux in RAW264.7 macrophages, thus reinforcing the hypothesis that 3-oxoC12:2-HSL signals through taste receptors. Furthermore, through a screening of all human bitter taste receptors, we identified six taste receptors that were activated in the presence of 3-oxo-C12:2-HSL. Some of them have numerous known ligands, such as TAS2R14 which can bind to more than 150 compounds, 
while others only have two known synthetic agonists like TAS2R13, according to the BitterDB database ${ }^{51}$. Advances in knowledge of BTR involvement in inflammatory pathways are needed to link our findings with AHLs signalling in immune cells.

In the present study, we restricted our investigation to the effect of 3-oxo-C12:2-HSL on host immune cells. One could imagine that 3-oxo-C12:2-HSL also exerts an effect on gut microbiota. Since we have previously shown that the absence of 3-oxo-C12:2-HSL is associated with differential representation of several bacterial taxa in fecal microbiota from IBD patients ${ }^{15}$, we could hypothesise that 3-oxo-C12:2-HSL participates in gut normobiosis. A study showed that the universal QS molecule, Al-2, was able to recapitulate gut microbiota following an in vivo antibiotic challenge. Al-2 was delivered by modified $E$. coli and increased Firmicutes proportions of the microbiota ${ }^{52}$. In the same way, the role of 3-oxo-C12:2-HSL in shaping gut microbiota needs to be examined. Its putative effects induced in the gut microbiota, which must be investigated, could be additive to its effects on host cells, leading to an overall beneficial effect in gut homeostasis.

Our work is part of an innovative approach highlighting the role of bacterial QS molecules in the gut apart from the interaction with a pathogen. Our results provide a first set of data on the interactions of the gastrointestinal tract with $A H L$ and we propose a model laying the foundation for the effect of 3-oxoC12:2-HSL on immune cells (Fig. 5). 3-oxo-C12:2-HSL is able to activate bitter taste receptors, resulting in calcium release from the endoplasmic reticulum. Besides, 3-oxo-C12:2-HSL prevents from the activation of the JAK-STAT pathways in activated macrophages by specifically preventing the phosphorylation of JAK1 and STAT1. Finally, the effect of 3-oxo-C12:2-HSL on immune cells highlights the role of this type of molecule in pathophysiology of puzzling diseases such as IBD where dysbiosis is involved in gut inflammation. Investigating QS molecules and their interkingdom effect therefore remains an unavoidable pursuit when searching for links between inflammation and gut microbiota.

\section{Methods}

\section{Cell culture}

RAW264.7 murine macrophage cells (ATCC, Manassas, Virginia, USA) were cultured between passage 12 and 25 in Dulbecco's modified Eagle's medium supplemented with $10 \%$ heat-inactivated fetal calf serum and $1 \% \mathrm{~L}$-glutamine (ThermoFisher Scientific). The macrophages were seeded at 35,000 cells per well in 12-well plates (Falcon) for ELISA experiments or 110,000 cells per well in 6-well plates (Falcon) for cell lysates, upon reaching $80-90 \%$ confluence after 3-day culture. Cells were maintained at $37^{\circ} \mathrm{C}$ with $5 \%$ CO2/air atmosphere and the media were changed every other day.

\section{Human Peripheral Blood Mononuclear Cell isolation}


Peripheral Blood Mononuclear Cells (PBMC) were extracted from whole blood of anonymous donor and collected by Etablissement Français du Sang of Paris, France.

PBMC were cultured in Roswell Park Memorial Institute medium (ThermoFisher Scientific) with 10\% heatinactivated fetal calf serum, $1 \%$ L-glutamine, $1 \%$ non-essential amino acids, $1 \%$ sodium pyruvate, penicillin $(100 \mathrm{lU} / \mathrm{ml})$, and streptomycin $(100 \mu \mathrm{g} / \mathrm{ml})$. Peripheral blood was harvested in EDTA tubes, blood was diluted with PBS 1:1, layered over Histopaque-1077® (Sigma) and centrifuged for 20 min at $400 \mathrm{~g}$ at room temperature. Extracted PBMC were washed twice with sterile PBS then centrifuged at $300 \mathrm{~g}$ for $5 \mathrm{~min}$ and resuspended in complete medium. For experiments, cells were seeded at 300,000 cells per well in $200 \mu \mathrm{L}$ of medium in 96-well plates (ThermoFisher Scientific).

\section{Cell Treatments}

Cells were exposed to $3-0 x 0-C 12: 2-H S L$ (synthesized as previously described ${ }^{15}$ or DMSO $0.1 \%$ as a control for different durations depending on the experiment, in the presence of the lactonase inhibitor 2hydroxyquinoline ( $100 \mu \mathrm{M}$, Sigma-Aldrich), in order to limit the hydrolysis of the AHL lactone ring, as described previously ${ }^{15,16}$.

In RAW264.7 cells, inflammation was triggered with LPS $(10 \mathrm{ng} / \mathrm{mL}$, Sigma) and IFNy $(10 \mathrm{U} / \mathrm{mL}$, R\&Dsystems) for $2 \mathrm{~h}$ or $6 \mathrm{~h}$, depending on the experiments. PBMC were exposed to LPS $(10 \mathrm{ng} / \mathrm{mL})$ and AHL for 24 hours. For TAS2R38 inhibition experiments, cells were exposed to TAS2R38 allosteric inhibitor probenecid (1 mM, Sigma) for one hour before adding AHLs and the inflammatory cocktail, without changing the media.

\section{Lactate dehydrogenase release}

Release of lactate dehydrogenase into the medium was measured to assess cellular toxicity. LDH concentration was measured using the Cytotoxicity Detection Kit (Roche, Boulogne-Billancourt, France) according to the manufacturer's instructions. The media were centrifuged $5 \mathrm{~min}$ at $250 \mathrm{~g}$ to remove any cells. $50 \mu \mathrm{L}$ of reaction mixture was added to $50 \mu \mathrm{L}$ of media supernatant in 96 -well plates. After $10 \mathrm{~min}$ at room temperature, $25 \mu \mathrm{L}$ of stop solution was added. Absorbance at $490 \mathrm{~nm}$ was determined with a microplate spectrometer (FLUOstar Omega; BMG Labtech). Results are expressed as absorbance arbitrary units.

\section{Enzyme-Linked Immunosorbent Assay}

At the end of cell treatment, the medium was centrifuged at $300 \mathrm{~g}$ for $5 \mathrm{~min}$ at $4^{\circ} \mathrm{C}$. Supernatant was frozen at $-80^{\circ} \mathrm{C}$ until ELISA assays. TNFa and IL-1 $\beta$ ELISA kits were purchased from R\&D systems and performed according to the manufacturer's instructions. Briefly, plates were coated with a capture antibody and incubated overnight. Wells were blocked using $1 \%$ bovine serum albumin-PBS solution 
(Sigma), then exposed to cell supernatants at room temperature for two hours. Wells were washed and incubated with the detection antibody for an additional two hours. After thorough washing, StreptavidinHRP solution was added for 20 min. Wells were washed, then incubated with TMB (Biolegend) solution

for $20 \mathrm{~min}$ and the reaction was stopped using $1 \mathrm{M} \mathrm{H}_{2} \mathrm{SO}_{4}$. Absorbance was measured by a spectrometer (FLUOstar Omega; BMG Labtech) at 450nm and the absorbance at $540 \mathrm{~nm}$ was subtracted.

\section{RNA extraction for RNA sequencing}

Cells were treated for 2 hours and then washed once with PBS before RNA extraction using RNeasy minikit (Qiagen), according to the manufacturer's instructions. Briefly, cells were lysed and 1 volume of ethanol was added. The sample was transferred into a spin column and centrifuged at 8,000 $\mathrm{g}$ for $15 \mathrm{sec}$. The column was washed several times and then eluted with water. RNA quality was assessed using NanoChip (Agilent) on a chip reader (Bioanalyzer 2100, Agilent)

\section{mRNA sequencing}

After extraction, total RNA were qualified with the AGILENT tapeStation 2200. Preparation of mRNA libraries was performed according to the manufacturer's recommendations (KAPA mRNA HyperPrep Kit from ROCHE). Final samples pooled into a library were sequenced on ILLUMINA Novaseq 6000, corresponding to $2 \times 28$ Millions of 100 bases reads per sample after demultiplexing.

\section{RNA-Seq data analysis workflow}

FastQC $v 0.11 .9^{53}$ was used for the quality control of raw paired-end fastq data set. Illumina's adapters were removed from reads using cutadapt $\mathrm{v} 2.10^{54}$. Trim_galore $\mathrm{v} 0.6 .4^{55}$ was used to trim bad quality bases (Phred < 20). Salmon v $1.4 .0^{56}$ was used to quantify paired-end reads against a mapping-based index built from the Ensembl GRCm38 transcript set for all samples. Downstream analysis was handled in R platform v4.0.2 $2^{57}$ and its add-on packages. Quantified transcripts were imported into $\mathrm{R}$ using the package tximport v1.16.1 ${ }^{58}$. Gene-level DESeqDataSet object was built from previously imported transcript level abundances using the package DESeq ${ }^{59}$ to perform the differential expression analysis. A pre-filtering was applied to remove genes with less than 5 counts in one sample. For visualisation, raw counts were transformed using the "variance stabilizing transformation" method implemented in the package DESeq2. For an overview of the variability among samples, Principal Component Analysis was performed on variance stabilizing transformation and normalised count dataset using the package ade4 $v 1.7-16^{60}$. Differential expression analysis was carried out by the DESeq function of the DESeq2 package. Briefly, the size factor was estimated for each sample, the dispersion was estimated for each gene, counts were fitted to a Negative Binomial Generalized Linear Model and Wald's test was conducted to test the difference in expression between biological conditions. The Benjamini-Hochberg method ${ }^{61}$ 
was used for controlling the False-discovery rate (FDR). Significance level was fixed at type I error alpha = 0.01. Volcano plots were used to display the results of differential analysis using the ggplot2 $v 3.3 .3^{62}$ package. All significant gene lists were annotated for enriched biological functions and pathways using the DAVID platform through the RDAVIDWebService v1.28.0 ${ }^{63}$ package for gene ontology ${ }^{64}$ and Kyoto Encyclopedia of Genes and Genomes terms ${ }^{65}$. Significant terms had adjusted p-value, according to the Benjamini-Hochberg method, below 0.05 . In addition to the traditional gene by gene analysis, count dataset was analysed at KEGG pathway level using the package Ensemble of Gene Set Enrichment Analysis (EGSEA) v1.16.066 that combines results from twelve algorithms to improve the biological relevance of pathways. Thus, significant pathways had adjusted p-value below 0.01 . Gene expression heatmaps were produced by the pheatmap $v 1.0 .12^{67}$ package. We used Venn diagrams to globally visualize the overlap between all significant genes as well as all significant pathways in the biological condition comparisons.

\section{Determination of protein levels by $\mathrm{Wes}^{\mathrm{TM}}$ capillary electrophoresis}

RAW264.7 cells were scraped in lysis buffer (20 mM Tris- $\mathrm{HCl}$, pH 7.4, 5 mM EDTA, $0.15 \mathrm{M} \mathrm{NaCl}, 1 \%$ Triton $\mathrm{X}-100,0.5 \%$ sodium deoxycholate) supplemented with protease inhibitor cocktail (Complete Mini; Roche, Boulogne-Billancourt, France) and phosphatase inhibitor cocktail (PhosSTOP, Sigma-Aldrich). Protein concentrations were determined using the BC Assay (Uptima/Interchim). Wes ${ }^{\mathrm{TM}}$ analyses (capillary electrophoresis system; ProteinSimple, San Jose, CA) were performed according to the manufacturer's recommendations, and adequate protein concentrations and antibody dilutions were determined in preliminary assays to allow optimal quantitative conditions. The microplates were loaded with $0.8 \mu \mathrm{g} / \mu \mathrm{L}$ protein, primary antibodies (as detailed below), and reagents provided by the manufacturer : anti-JAK1 (1:25; Biotechne MAB4260), anti-Phospho-JAK1 (1:100 ; Invitrogen 44422G), anti-JAK2 (1:13; Cell Signalling, 3230), anti-Phospho-JAK2 (1:13; Cell Signalling 3776), anti-STAT1 (1:50; Cell Signalling 9172), anti-Phospho-STAT1 (1:40 ; Biotechne AF2894), anti-Actin (1:1000; Millipore MAB1501R). Data were analysed using Compass for SW3.1 software (ProteinSimple). Protein levels were determined by chemiluminescence signal (AUC) and Phospsho-Protein levels were normalized to the total level of each protein of interest, after verification that actin levels do not differ between conditions.

\section{Bitter Taste Receptors Screening}

Bitter taste receptor cDNA were cloned into the pcDNA4 mammalian expression vector (Invitrogen) using full coding receptor sequences reported on UniProt database. The synthetic constructs were codon optimized (Genewiz) and combine the first 45 amino acids of rat somatostatin type 3 receptor at the amino terminus to improve plasma membrane targeting of the receptors in the heterologous system ${ }^{68}$. The FLAG epitope was added to the carboxy terminus without interfering with receptor functionality and 
can be used for immunocytochemistry. Calcium-mobilization assays were performed using human embryonic kidney cells (HEK293T) stably transfected with chimeric Ga16gust44 protein ${ }^{69}$. Cells were seeded in black, poly-D-lysine coated 96 -well plates at a density of 35000 cells $/ 100 \mu \mathrm{L}$ in high glucose DMEM supplemented with $10 \%$ dialysed fetal bovine serum and $1 \%$ penicillin/streptomycin. Twenty-four hours later, the cells were transiently co-transfected using Lipofectamine 2000 (Life Technologies) with one of the TAS2R synthetic optimized vectors or empty expression plasmid, as a negative control, and the pCMV-GCaMP5G construct (Addgene \#31788) coding for a genetically encoded calcium indicator ${ }^{70,71}$.

Twenty-four hours after transfection, cells were washed twice with $\mathrm{C} 1$ buffer $(130 \mathrm{mM} \mathrm{NaCl}, 5 \mathrm{mM} \mathrm{KCl}, 10$ $\mathrm{mM}$ HEPES, $2 \mathrm{mM} \mathrm{CaCl} 2,5 \mathrm{mM}$ sodium pyruvate, $\mathrm{pH}$ 7.4). 3-oxo-C12:2-HSL was solved in DMSO and further diluted in $\mathrm{C} 1$ buffer. Next, the cells were placed in a fluorometric imaging plate reader (Flexstation ${ }^{\circledR} 3$, Molecular Devices) and stimulated with automatic injection of increasing concentrations of HSLs. Calcium responses leading to increase in fluorescence were monitored at $510 \mathrm{~nm}$ after excitation at $488 \mathrm{~nm}$. Experiments were performed in duplicates and repeated at least four times. The recorded calcium levels of each wells receiving the same stimulus were averaged, response of mocktransfected cells were subtracted from receptor-transfected cells and net signals were normalized to background fluorescence (F/F0, F0 fluorescence light before stimulus application). The resulting doseresponse curves of the averaged fluorescent signal amplitudes against the logarithm of the agonist concentration were fitted using Sigma Plot software. For data analysis, the four-parameter logistic equation $\left[\mathrm{f}(\mathrm{x})=\min +(\max -\mathrm{min}) /\left(1+\left(\mathrm{x} / \mathrm{EC}_{50}\right) \mathrm{nH}\right)\right]$ was used to calculate the half-maximal effective concentrations ( $\mathrm{EC}_{50}$ values).

\section{RNA extraction and RT-qPCR}

Total RNA was extracted from RAW264.7 cells using TRIzol (Invitrogen) according to the manufacturer's instructions. Reverse Transcription (RT) was performed with $1 \mu \mathrm{g}$ RNA using LunaScript@ RT SuperMix Kit (NewEngland Biolabs). Semi-quantitative real-time PCR was performed with the Mx3000P Stratagene system using SYBR Green (Applied Biosystems) according to the manufacturer's procedures. Cyclophilin was used as the reference gene. After amplification, $\mathrm{Ct}$ were determined. Sequences of the oligonucleotide primers used are reported in table 1.

\section{Table 1}

Sequence of the primers used in this study 


\begin{tabular}{|lll|}
\hline Target gene (Protein) & Forward primer & Reverse primer \\
\hline Tnf $($ TNFa $)$ & CCAGACCCTCACACTGAGATC & CACTTGGTGGTTTGCTACGAC \\
\hline I1b $(\mathrm{IL}-1 \beta)$ & AGTTGACGGACCCCAAAAG & AGCTGGATGCTCTCATCAGG \\
\hline$\| 10(\mathrm{IL}-10)$ & CTGGACAACATACTGCTAACC & GGGCATCACTTCTACCAGGTA \\
\hline Ppib (Cyclophilin B) & GCCTTAGCTACAGGAGAGAA & TTTCCTCCTGTGCCATCTC \\
\hline
\end{tabular}

\section{Intracellular calcium assay}

RAW264.7 cells were seeded in black, clear-bottom 96-well plates (Costar) at 20,000 cells per well. The next day, release of intracellular $\mathrm{Ca}^{2+}$ was measured using Fluo-4 (Fluo-4 Direct Calcium Assay; Life Technologies) according to the manufacturer's instructions. Medium was discarded and $100 \mu \mathrm{L}$ of $1 \mathrm{X}$ Fluo-4 dye was incubated at $37^{\circ} \mathrm{C}$ for $1 \mathrm{~h}$ in $5 \% \mathrm{CO}_{2}$, protected from light. Then, basal fluorescence was measured using an fluorospectrometer (FLUOstar Omega; BMG Labtech), excitation at $485 \mathrm{~nm}$ and emission at $520 \mathrm{~nm}$. Molecules of interest were then added with an electronic pipette; thapsigargin (10 $\mu \mathrm{M}$, Sigma) as a positive control, 3-oxo-C12:2-HSL $(1,000 \mu \mathrm{M})$ or DMSO $0.5 \%$; with 8 replicates per conditions for every experiment. The fluorescence signal was immediately measured every 80 seconds for $2 \mathrm{~h}$. Results are expressed as a ratio of the fluorescence $\mathrm{x}$ times over the basal fluorescence (F/F0).

\section{Quantification and statistical analysis}

Data are expressed as means \pm standard error of the mean (SEM) unless otherwise indicated. The number of independent experiments and replicates are indicated in the figure captions. Figures and statistical analyses were performed with Graphpad ${ }^{\circledR}$ Prism 8.0 (Ritme Informatique, Paris, France). Normal distribution was tested using Shapiro-Wilk test. When normal distribution was confirmed, statistical differences between the means were assessed by ANOVA or t-test otherwise by a nonparametric Kruskal-Wallis test. Post-test are indicated in the figure captions. For all results, $p<0.05$ was

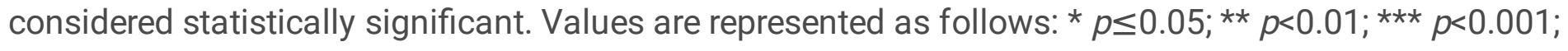
$p<0.0001$.

\section{Declarations}

\section{Data Availability Statement:}

The datasets generated and/or analysed during the current study are available from the corresponding author upon reasonable request.

\section{Ethics declarations:}


Peripheral Blood Mononuclear Cells (PBMC) were extracted from whole blood of an anonymous donor and collected by Etablissement Français du Sang of Paris, France. Informed consent was obtained from all participants.

\section{Acknowledgments}

This research was funded by Fondation pour la Recherche Médicale, grant number EQU202003010171 and by the Association François Aupetit (projects research on AHLs 2019 and 2021). G. Coquant was the recipient of a fellowship from Fondation pour la Recherche Médicale FRM EC0201806006843. D. Aguanno was the recipient of a fellowship from CORDDIM, lle-de-France region. The authors acknowledge the Association François Aupetit for its unwavering support. The authors thank Yannick Marie and his team at the Plateforme de Genotypage/Séquençage of the Institut du Cerveau et de la Moelle épinière for the RNA sequencing.

\section{Author contributions}

GC, ST, PS conceived the experiments; GC, DA, L. Brot, CB, JD, MM and NR conducted the experiments and analysis; LDS, J-PG, VC, ST, PS, L. Briand supervised the work, GC and PS wrote the main manuscript text; GC prepared all the figures. Funding Acquisition: ST, PS. All authors reviewed the manuscript.

\section{Competing Interests Statement}

P Seksik declares speaker and consulting fees from Takeda, Merck MSD, Biocodex, Ferring, Janssen, Amgen, Pfizer, Pileje, Fresenius Kabi and Abbvie but has no conflict of interest linked to this work.

\section{References}

1. Maaser, C. et al. ECCO-ESGAR Guideline for Diagnostic Assessment in IBD Part 1: Initial diagnosis, monitoring of known IBD, detection of complications. Journal of Crohn's and Colitis 13, 144-164K (2019).

2. Burisch, J., Jess, T., Martinato, M. \& Lakatos, P. L. The burden of inflammatory bowel disease in Europe. Journal of Crohn's and Colitis 7, 322-337 (2013).

3. Hernandez-Rocha, C. \& Vande Casteele, N. Corrigendum to 'JAK inhibitors: Current position in treatment strategies for use in inflammatory bowel disease'. Current Opinion in Pharmacology 58, 68 (2021).

4. Hashash, J. Biologic monotherapy versus combination therapy with immunomodulators in the induction and maintenance of remission of Crohn's disease and ulcerative colitis. aog (2021) doi:10.20524/aog.2021.0645. 
5. Sokol, H. et al. Faecalibacterium prausnitzii is an anti-inflammatory commensal bacterium identified by gut microbiota analysis of Crohn disease patients. Proc Natl Acad Sci U S A 105, 16731-16736 (2008).

6. IBDMDB Investigators et al. Multi-omics of the gut microbial ecosystem in inflammatory bowel diseases. Nature 569, 655-662 (2019).

7. Sokol, H. \& Seksik, P. The intestinal microbiota in inflammatory bowel diseases: time to connect with the host: Current Opinion in Gastroenterology 26, 327-331 (2010).

8. Cheng, Y.-W. \& Fischer, M. Fecal Microbiota Transplantation for Ulcerative Colitis. Are We Ready for Primetime? Gastroenterology Clinics of North America 49, 739-752 (2020).

9. Saint-Antoine IBD Network et al. Fecal microbiota transplantation to maintain remission in Crohn's disease: a pilot randomized controlled study. Microbiome 8, 12 (2020).

10. Lavelle, A. \& Sokol, H. Gut microbiota-derived metabolites as key actors in inflammatory bowel disease. Nature Reviews Gastroenterology \& Hepatology (2020) doi:10.1038/s41575-019-0258-z.

11. Duboc, H. et al. Connecting dysbiosis, bile-acid dysmetabolism and gut inflammation in inflammatory bowel diseases. Gut 62, 531-539 (2013).

12. Lamas, B. et al. CARD9 impacts colitis by altering gut microbiota metabolism of tryptophan into aryl hydrocarbon receptor ligands. Nat Med 22, 598-605 (2016).

13. Quevrain, E. et al. Identification of an anti-inflammatory protein from Faecalibacterium prausnitzii, a commensal bacterium deficient in Crohn's disease. Gut (2016).

14. Coquant, G., Grill, J.-P. \& Seksik, P. Impact of N-Acyl-Homoserine Lactones, Quorum Sensing Molecules, on Gut Immunity. Front. Immunol. 11, 1827 (2020).

15. Landman, C. et al. Inter-kingdom effect on epithelial cells of the N-Acyl homoserine lactone 3-oxoC12:2, a major quorum-sensing molecule from gut microbiota. PLOS ONE 13, e0202587 (2018).

16. Aguanno, D. et al. The intestinal quorum sensing 3-oxo-C12:2 Acyl homoserine lactone limits cytokine-induced tight junction disruption. Tissue Barriers 1832877 (2020) doi:10.1080/21688370.2020.1832877.

17. Moreira Lopes, T. C., Mosser, D. M. \& Gonçalves, R. Macrophage polarization in intestinal inflammation and gut homeostasis. Inflamm. Res. 69, 1163-1172 (2020).

18. Bryan, A. et al. Human transcriptome analysis reveals a potential role for active transport in the metabolism of Pseudomonas aeruginosa autoinducers. Microbes and Infection 12, 1042-1050 (2010).

19. Yeshi, K. et al. Revisiting Inflammatory Bowel Disease: Pathology, Treatments, Challenges and Emerging Therapeutics Including Drug Leads from Natural Products. JCM 9, 1273 (2020).

20. Danese, S., Argollo, M., Le Berre, C. \& Peyrin-Biroulet, L. JAK selectivity for inflammatory bowel disease treatment: does it clinically matter? Gut 68, 1893-1899 (2019).

21. Maurer, S. et al. Tasting Pseudomonas aeruginosa Biofilms: Human Neutrophils Express the Bitter Receptor T2R38 as Sensor for the Quorum Sensing Molecule N-(3-Oxododecanoyl)-I-Homoserine 
Lactone. Frontiers in Immunology 6, (2015).

22. Jaggupilli, A., Singh, N., Jesus, V. C. D., Duan, K. \& Chelikani, P. Characterization of the Binding Sites for Bacterial Acyl Homoserine Lactones (AHLs) on Human Bitter Taste Receptors (T2Rs). ACS Infect. Dis. 4, 1146-1156 (2018).

23. Lu, P., Zhang, C.-H., Lifshitz, L. M. \& ZhuGe, R. Extraoral bitter taste receptors in health and disease. Journal of General Physiology 149, 181-197 (2017).

24. Jeon, T.-I., Seo, Y.-K. \& Osborne, T. F. Gut Bitter Taste Receptor Signaling Induces ABCB1 through a Mechanism Involving CCK. Biochem J 438, 33-37 (2011).

25. Latorre, R. et al. Expression of the Bitter Taste Receptor, T2R38, in Enteroendocrine Cells of the Colonic Mucosa of Overweight/Obese vs. Lean Subjects. PLoS ONE 11, e0147468 (2016).

26. Turner, A. et al. Interactions between taste receptors and the gastrointestinal microbiome in inflammatory bowel disease. Journal of Nutrition \& Intermediary Metabolism 18, 100106 (2019).

27. Yu, Y. et al. Berberine induces GLP-1 secretion through activation of bitter taste receptor pathways. Biochemical Pharmacology 97, 173-177 (2015).

28. Tran, H. T. T., Herz, C., Ruf, P., Stetter, R. \& Lamy, E. Human T2R38 Bitter Taste Receptor Expression in Resting and Activated Lymphocytes. Frontiers in Immunology 9, (2018).

29. Verbeurgt, C. et al. The human bitter taste receptor T2R38 is broadly tuned for bacterial compounds. PLOS ONE 12, e0181302 (2017).

30. Greene, T. A. et al. Probenecid Inhibits the Human Bitter Taste Receptor TAS2R16 and Suppresses Bitter Perception of Salicin. PLoS ONE 6, e20123 (2011).

31. Wölfle, U. et al. Expression and Functional Activity of the Human Bitter Taste Receptor TAS2R38 in Human Placental Tissues and JEG-3 Cells. Molecules 21, 306 (2016).

32. Meyerhof, W. et al. The Molecular Receptive Ranges of Human TAS2R Bitter Taste Receptors. Chemical Senses 35, 157-170 (2010).

33. Telford, G. et al. The Pseudomonas aeruginosa quorum-sensing signal molecule N-(3oxododecanoyl)-L-homoserine lactone has immunomodulatory activity. Infect Immun 66, 36-42 (1998).

34. Glucksam-Galnoy, Y. et al. The Bacterial Quorum-Sensing Signal Molecule N-3-Oxo-Dodecanoyl-LHomoserine Lactone Reciprocally Modulates Pro- and Anti-Inflammatory Cytokines in Activated Macrophages. The Journal of Immunology 191, 337-344 (2013).

35. Kravchenko, V. V. et al. Modulation of Gene Expression via Disruption of NF-kB Signaling by a Bacterial Small Molecule. Science 321, 259 (2008).

36. Li, Y. et al. N-3-(oxododecanoyl)-L-homoserine lactone promotes the induction of regulatory T-cells by preventing human dendritic cell maturation. Exp Biol Med (Maywood) 240, 896-903 (2015).

37. Ritchie, A. J. et al. The Pseudomonas aeruginosa quorum-sensing molecule N-3-(oxododecanoyl)-Lhomoserine lactone inhibits T-cell differentiation and cytokine production by a mechanism involving an early step in T-cell activation. Infection and immunity 73, 1648-55 (2005). 
38. Hooi, D. S. W., Bycroft, B. W., Chhabra, S. R., Williams, P. \& Pritchard, D. I. Differential Immune Modulatory Activity of Pseudomonas aeruginosa Quorum-Sensing Signal Molecules. Infection and Immunity 72, 6463-6470 (2004).

39. Peyrottes, A. et al. Anti-Inflammatory Effects of Analogues of N-Acyl Homoserine Lactones on Eukaryotic Cells. Int. J. Mol. Sci. 19 (2020).

40. Isidro, R. A. \& Appleyard, C. B. Colonic macrophage polarization in homeostasis, inflammation, and cancer. 15.

41. Darnell, J., Kerr, I. \& Stark, G. Jak-STAT pathways and transcriptional activation in response to IFNs and other extracellular signaling proteins. Science 264, 1415-1421 (1994).

42. Held, T. K., Weihua, X., Yuan, L., Kalvakolanu, D. V. \& Cross, A. S. Gamma Interferon Augments Macrophage Activation by Lipopolysaccharide by Two Distinct Mechanisms, at the Signal Transduction Level and via an Autocrine Mechanism Involving Tumor Necrosis Factor Alpha and Interleukin-1. Infect Immun 67, 206-212 (1999).

43. Orecchioni, M., Ghosheh, Y., Pramod, A. B. \& Ley, K. Macrophage Polarization: Different Gene Signatures in M1(LPS+) vs. Classically and M2(LPS-) vs. Alternatively Activated Macrophages. Front. Immunol. 10, 1084 (2019).

44. Li, L., Hooi, D., Chhabra, S. R., Pritchard, D. \& Shaw, P. E. Bacterial N-acylhomoserine lactone-induced apoptosis in breast carcinoma cells correlated with down-modulation of STAT3. Oncogene 23, 4894-4902 (2004).

45. Odenwald, M. A. \& Turner, J. R. The intestinal epithelial barrier: a therapeutic target? Nature Reviews Gastroenterology \& Hepatology 14, 9-21 (2016).

46. Moura-Alves, P. et al. Host monitoring of quorum sensing during Pseudomonas aeruginosa infection. Science (New York, N.Y.) 366, (2019).

47. Cooley, M. A., Whittall, C. \& Rolph, M. S. Pseudomonas signal molecule 3-oxo-C12-homoserine lactone interferes with binding of rosiglitazone to human PPARy. Microbes and Infection 12, 231237 (2010).

48. Jahoor, A. et al. Peroxisome Proliferator-Activated Receptors Mediate Host Cell Proinflammatory Responses to Pseudomonas aeruginosa Autoinducer. Journal of Bacteriology 190, 4408-4415 (2008).

49. Lee, R. J. et al. T2R38 taste receptor polymorphisms underlie susceptibility to upper respiratory infection. J. Clin. Invest. 122, 4145-4159 (2012).

50. Gaida, M. M. et al. Expression of the bitter receptor T2R38 in pancreatic cancer: localization in lipid droplets and activation by a bacteria-derived quorum-sensing molecule. Oncotarget 7, (2016).

51. Dagan-Wiener, A. et al. BitterDB: taste ligands and receptors database in 2019. Nucleic Acids Research 47, D1179-D1185 (2019).

52. Thompson, J. A., Oliveira, R. A., Djukovic, A., Ubeda, C. \& Xavier, K. B. Manipulation of the Quorum Sensing Signal Al-2 Affects the Antibiotic-Treated Gut Microbiota. Cell Reports 10, 1861-1871 (2015). 
53. Andrews, S. fastQC. (Babraham Institute, 2019).

54. Martin, M. Cutadapt removes adapter sequences from high-throughput sequencing reads. EMBnet $j$. $17,10(2011)$.

55. Krueger, F. Trim Galore. (Babraham Institute, 2019).

56. Patro, R., Duggal, G., Love, M. I., Irizarry, R. A. \& Kingsford, C. Salmon provides fast and bias-aware quantification of transcript expression. Nat Methods 14, 417-419 (2017).

57. Team, R. D. C. R: A Language and Environment for Statistical Computing. (2020).

58. Soneson, C., Love, M. I. \& Robinson, M. D. Differential analyses for RNA-seq: transcript-level estimates improve gene-level inferences. F1000Res 4, 1521 (2015).

59. Love, M. I., Huber, W. \& Anders, S. Moderated estimation of fold change and dispersion for RNA-seq data with DESeq2. Genome Biology 15, (2014).

60. Dray, S. \& Dufour, A. B. The ade4 Package: Implementing the Duality Diagram for Ecologists. Journal of Statistical Software 22, (2007).

61. Benjamini, Y. \& Hochberg, Y. Controlling the False Discovery Rate: A Practical and Powerful Approach to Multiple Testing. Journal of the Royal Statistical Society. Series B (Methodological) 57, 289-300 (1995).

62. Wickham, H. ggplot2: Elegant Graphics for Data Analysis. (Springer-Verlag New York, 2016).

63. Fresno, C. \& Fernandez, E. A. RDAVIDWebService: a versatile R interface to DAVID. Bioinformatics 29, 2810-2811 (2013).

64. Ashburner, M. et al. Gene ontology: tool for the unification of biology. The Gene Ontology Consortium. Nat. Genet. 25, 25-29 (2000).

65. Kanehisa, M. \& Goto, S. KEGG: kyoto encyclopedia of genes and genomes. Nucleic Acids Res. 28, 27-30 (2000).

66. Alhamdoosh, M. et al. Combining multiple tools outperforms individual methods in gene set enrichment analyses. Bioinformatics btw623 (2016) doi:10.1093/bioinformatics/btw623.

67. Kolde, R. pheatmap: Pretty Heatmaps. (2019).

68. Meyerhof, W. et al. The Molecular Receptive Ranges of Human TAS2R Bitter Taste Receptors. Chemical Senses 35, 157-170 (2009).

69. Raliou, M. et al. Human Genetic Polymorphisms in T1R1 and T1R3 Taste Receptor Subunits Affect Their Function. Chemical Senses 36, 527-537 (2011).

70. Akerboom, J. et al. Optimization of a GCaMP calcium indicator for neural activity imaging. $J$ Neurosci 32, 13819-13840 (2012).

71. Cai, B. et al. A Cell-Based Functional Assay Using a Green Fluorescent Protein-Based Calcium Indicator dCys-GCaMP. ASSAY and Drug Development Technologies 12, 342-351 (2014).

\section{Figures}




\section{Figure 1}

3-oxo-C12:2-HSL decreases pro-inflammatory cytokine secretion by immune cells Secreted TNFa (a) and IL-1b (b) levels measured by ELISA from supernatant of RAW264.7 macrophages were stimulated with LPS and IFNg in absence (control) or presence of $50 \mu \mathrm{M}$ 3-oxo-C12:2-HSL for 6 hours. Unpaired t-test, *

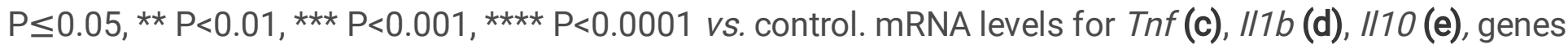
measured by RT-qPCR in RAW264.7 macrophages were stimulated with LPS and IFNg in absence (control) or presence of $50 \mu \mathrm{M}$ 3-oxo-C12:2-HSL for 2 hours. Results are expressed in arbitrary units as a ratio of the target gene to cyclophilin (Ppib) used as housekeeping gene. Unpaired t-test, * $P \leq 0.05$, **

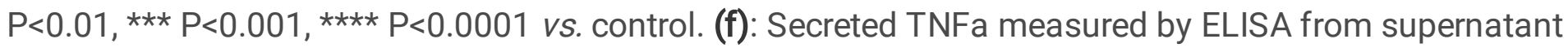

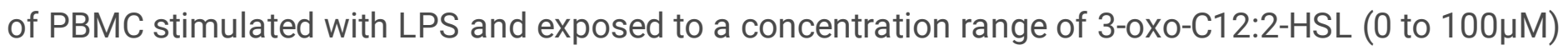
with LPS $(10 \mathrm{ng} / \mathrm{mL})$. Results are expressed as mean \pm SEM of triplicates from 3 independent experiments). One Way ANOVA Dunnett's post-test. 3oC12:2 stands for 3-oxo-C12:2-HSL.

\section{Figure 2}

\section{3-oxo-C12:2-HSL modulates gene expression in RAW264.7 macrophage cells}

Cells were stimulated with LPS and IFNg in absence (control) or in presence of $50 \mu \mathrm{M}$ 3-oxo-C12:2-HSL for 2 hours (a): Volcano plot of differentially expressed genes between the activated cells cultured in absence and in presence of 3-oxo-C12:2-HSL. Blue, grey and red dots represent down regulated, not significantly regulated and up regulated genes respectively. (b) Significant KEGG pathways involved in the inflammation process and differentially modulated by 3-oxo-C12:2-HSL were identified by EGSA method ( $p$ value $<0.01$ ). Red and green bars represent down-regulated and up-regulated pathways respectively. All significant modulated pathways are displayed in Supplementary Figure 2. (c) Significant Gene Ontology (GO) biology processes enriched by differentiated expressed genes modulated by 3-oxoC12:2-HSL. Terms were annotated using the Database for Annotation, Visualization and Integrated Discovery (DAVID). Red and green bars represent down-regulated and up-regulated pathways respectively. (GO: Gene Ontology, KEGG: Kyoto Encyclopedia of Genes and Genomes)

\section{Figure 3}


Cells were stimulated with LPS and IFNg in absence (control) or in presence of $50 \mu \mathrm{M}$ 3-oxo-C12:2-HSL for 2 hours. The levels of phosphorylated proteins P-JAK1 (a), STAT1 (b), JAK2 (c), STAT3 (d) were normalized to their respective unphosphorylated forms. Results are expressed as mean \pm SEM from 3

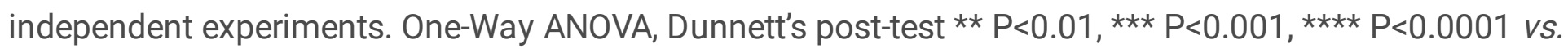
control. (e) Reconstructed images from automated capillary electrophoresis Simple Western analysis of protein levels and actin used as housekeeping protein are displayed. They are based on the area under the chemiluminescence signal curve obtained for one experiment, representative of 4 independent experiments performed in duplicates. Molecular markers are indicated on the left $(\mathrm{kDa})$. Reconstructed gels are cropped on the band of interest, complete reconstructed gels are available in supplementary figure 3. 3oC12:2 stands for 3-oxo-C12:2-HSL.

\section{Figure 4}

3-oxo-C12:2-HSL interacts with bitter taste receptors. (a): RAW264.7 macrophages were pre-treated or not with $1 \mathrm{mM}$ Probenecid, an allosteric inhibitor of the bitter taste receptor TAS2R138 one hour before their stimulation with LPS and IFNy in absence (control) or in presence of 3-oxo-C12:2-HSL for six hours. Mean \pm SEM from 3 independent experiments performed in triplicate, Two Way ANOVA Tukey's post-test (b) Calcium response in HEK293T cells expressing reporter human bitter taste receptor exposed to several

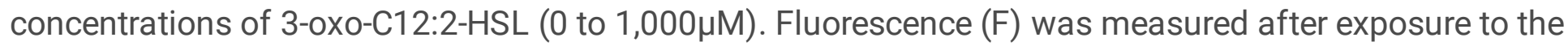
$\mathrm{AHL}$ and normalized to basal fluorescence (F0). Mean \pm SEM from 3 independent experiments performed in triplicate (c): Intracellular calcium flux in RAW264.7 cells exposed to 1,000 $\mu \mathrm{M}$ 3-oxo-C12:2-HSL or 10 $\mu \mathrm{M}$ thapsigargin as a positive control or DMSO $(0.5 \%)$ as a negative control. Fluorescence $(\mathrm{F})$ monintoring the increase in intracellular concentration of calcium was measured for two hours and normalized to basal fluorescence (F0). Each curve is representative of 3 independent experiments of 8 replicates. (d): Quantification of the slope of the curves displayed in (c). Values are mean \pm SEM of 3 independent experiments (8 replicates each). Two Way ANOVA Tukey's post-test. For A and D: * $P \leq 0.05$,

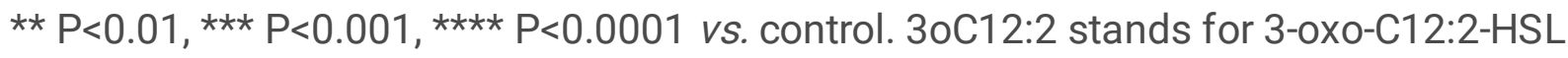

\section{Figure 5}

\section{Proposed mechanisms of 3-oxo-C12:2-HSL effects on inflammation}


In immune cells, 3-oxo-C12:2-HSL is able to activate bitter taste receptors, which are G-protein coupled receptors, triggering a signalling cascade resulting in the release of calcium from the endoplasmic reticulum. In addition, in murine activated macrophages the presence of 3-oxo-C12:2-HSL attenuates the activation of the JAK-STAT signalling pathways, by specifically preventing JAK1 and STAT1 phosphorylation. This leads to a decrease in pro-inflammatory cytokine secretions and an overall reduced inflammatory response. Part of the effects of 3-oxo-C12:2-HSL on inflammatory response is dependent on bitter taste receptors. Created with BioRender.com.

\section{Supplementary Files}

This is a list of supplementary files associated with this preprint. Click to download.

- CoquantetalSupp.docx 